\title{
Detailed study of macrobending effects in a wide transmission bandwidth hollow-core photonic bandgap fiber
}

\author{
Y. Chen", S.R. Sandoghchi, E. Numkam, T.D. Bradley, J.R. Hayes, N.V. Wheeler, G. Jasion, \\ D.R. Gray, F. Poletti, M.N. Petrovich, D.J. Richardson \\ Optoelectronics Research Centre, University of Southampton, Highfield, Southampton, SO17 1BJ, \\ United Kingdom. \\ Author e-mail address: (*) yclm12@soton.ac.uk
}

\begin{abstract}
We study in detail the macrobending effects in a wide transmission bandwidth ( 200nm) 19 cell hollow-core photonic bandgap fiber operating at 1550nm. Our results indicate low bend sensitivity over a $\sim 130 \mathrm{~nm}$ wide interval within the transmission window, with negligible loss $(<0.1 \mathrm{~dB})$ for bending radii down to $5 \mathrm{~mm}$. The "red shift" and "blue shift" of the bandgap edge have been observed at the short and long wavelength edges, respectively. The cutoff wavelengths where air-guiding modes stop guiding can be extracted from the bending loss spectra, which matches well with the simulated effective refractive index map of such fiber.
\end{abstract}

Keywords: Microstructured fibers, Hollow-core photonic bandgap fibers, Bending loss

\section{INTRODUCTION}

Hollow-core photonic bandgap fibers (HC-PBGFs) have attracted interest ever since their first appearance, as the guidance in an air core provides extremely low optical nonlinearity, the ultimate low-latency, potential for low loss, as well as an ideal platform for extremely efficient interaction between light and gases ${ }^{1}$. In recent years, research has focused on fiber development, with significant improvements achieved in terms of increasing the operating bandwidth, understanding and controlling the modal properties and scaling up the longitudinally uniform fiber length ${ }^{2,3}$. Several high performance HC-PBGFs have been demonstrated so far for a range of applications. Among them, for the reasons of low latency, low nonlinearity and potential (not yet demonstrated experimentally) to outperform conventional fibers in terms of transmission loss, these fibers are of great interest for tele-communication and other time-sensitive applications, such as intra and inter data center interconnection, high performance computing and bespoke data network for financial sectors $^{1}$. In order for HC-PBGFs to be deployed for application trials of realistic scale and situation, a number of fiber properties often overlooked, such as bend related loss and mode coupling effects, should be thoroughly investigated. Macrobending effect, for instance, is one of such important properties. More importantly, it is relevant not just to telecom/datacom applications but also to sensing applications, where the ability to achieve a compact device footprint by tightly coiling the fiber is often of paramount importance. Previous studies on macrobending effects used a high loss fiber and only the "red shift" feature was observed ${ }^{4}$. Here we present a detailed study of macrobending effects on a stateof-the-art HC-PBGF with combination of extremely wide transmission bandwidth $(>200 \mathrm{~nm})$ and low loss $(\sim 5 \mathrm{~dB} / \mathrm{km})$, and excellent longitudinal uniformity ${ }^{5}$.

Our measurements indicate that the fiber is substantially bend-insensitive over a bandwidth region of $\sim 130 \mathrm{~nm}$ at the center of the transmission bandgap, with negligible loss $(<0.1 \mathrm{~dB})$ for bending radii down to $5 \mathrm{~mm}$ and 30 turns. At wavelengths near the bandgap edges and in proximity of surface mode anti-crossings, the fiber shows more obvious bend sensitivity, which we explain in terms of bend-induced coupling to lossier higher-order/surface/cladding modes. As will be shown in the following, such increased mode coupling gives rise to a number of different effects. Bend-induced coupling to cladding modes and/or surface modes causes a substantial shift of the short wavelength edge to longer wavelengths and to a less pronounced shift of the long wavelength edge to shorter wavelengths. The combination of these two effects results in a narrowing of the transmission bandgap of the fiber. The effects of air-guiding modes' cutoff and higher order core-guiding modes subjecting to higher bending loss produce steps corresponding to the cutoff points of such modes. 


\section{DESCRIPTION OF HC-PBGF UNDER TEST}

The HC-PBGF utilized in this study was described in an earlier work ${ }^{5}$. This fiber has a 19 cell core and $5 \frac{1}{2}$ rings of cladding holes. It has a $\sim 153 \mu \mathrm{m}$ outer diameter, $\sim 30 \mu \mathrm{m}$ core diameter, $6.2 \mu \mathrm{m}$ hole-to-hole spacing $(\Lambda)$, a relative hole size $(\mathrm{d} / \Lambda)$ of $\sim 0.99$ and average cladding strut thickness of just $\sim 50 \mathrm{~nm}$. The mode field diameter of this fiber at $1550 \mathrm{~nm}$ is estimated to be $\sim 22 \mu \mathrm{m}$. This means that the core diameter (hence the corresponding mode field diameter) of this fiber almost double the size reported in an earlier study by Hansen et $\mathrm{al}^{4}$. A Scanning Electron Microscope (SEM) image of the fiber is shown in Fig. 1(a). The spectral loss of the fiber, determined via a cutback measurement, is shown in Fig. 2 (b). The lowest loss value is around $5.2 \mathrm{~dB} / \mathrm{km}$ at $1560 \mathrm{~nm}$. It is worth noting that this fiber has a very flat loss spectrum and a $3 \mathrm{~dB}$ bandwidth (wavelength interval over which the loss is less than twice the minimum loss) exceeding $200 \mathrm{~nm}$ (this was determined by another cutback measurement on a short section of this long fiber where the measurement was not limited by source power and Optical Spectrum Analyzer (OSA) dynamic range). Outside the low loss region, the fiber shows transmission with higher loss from $\sim 1225$ to $1725 \mathrm{~nm}$, with two broad loss peaks due to groups of surface modes at around 1350 and $1425 \mathrm{~nm}$; these can be easily identified in the spectral transmission recorded in "loose" condition shown in Fig 2(a). In order to assess the longitudinal uniformity of this fiber, a novel side scattering measurement was carried out for the whole fiber length 6 . The result is shown in Fig 3 (c), in which a flat slope corresponding to $\sim 5 \mathrm{~dB} / \mathrm{km}$ loss is observed and only minor deviations and very small scattering events, demonstrating that the fiber structure is highly uniform along the full length.

For the present study we used samples taken from this $11 \mathrm{~km}$ long fiber. The various measurements carried out and described in the following section used approximately $60 \mathrm{~m}$ of the fiber. By virtue of the excellent longitudinal uniformity of the fiber, we are confident that the results obtained reliably describe the properties along the full length.
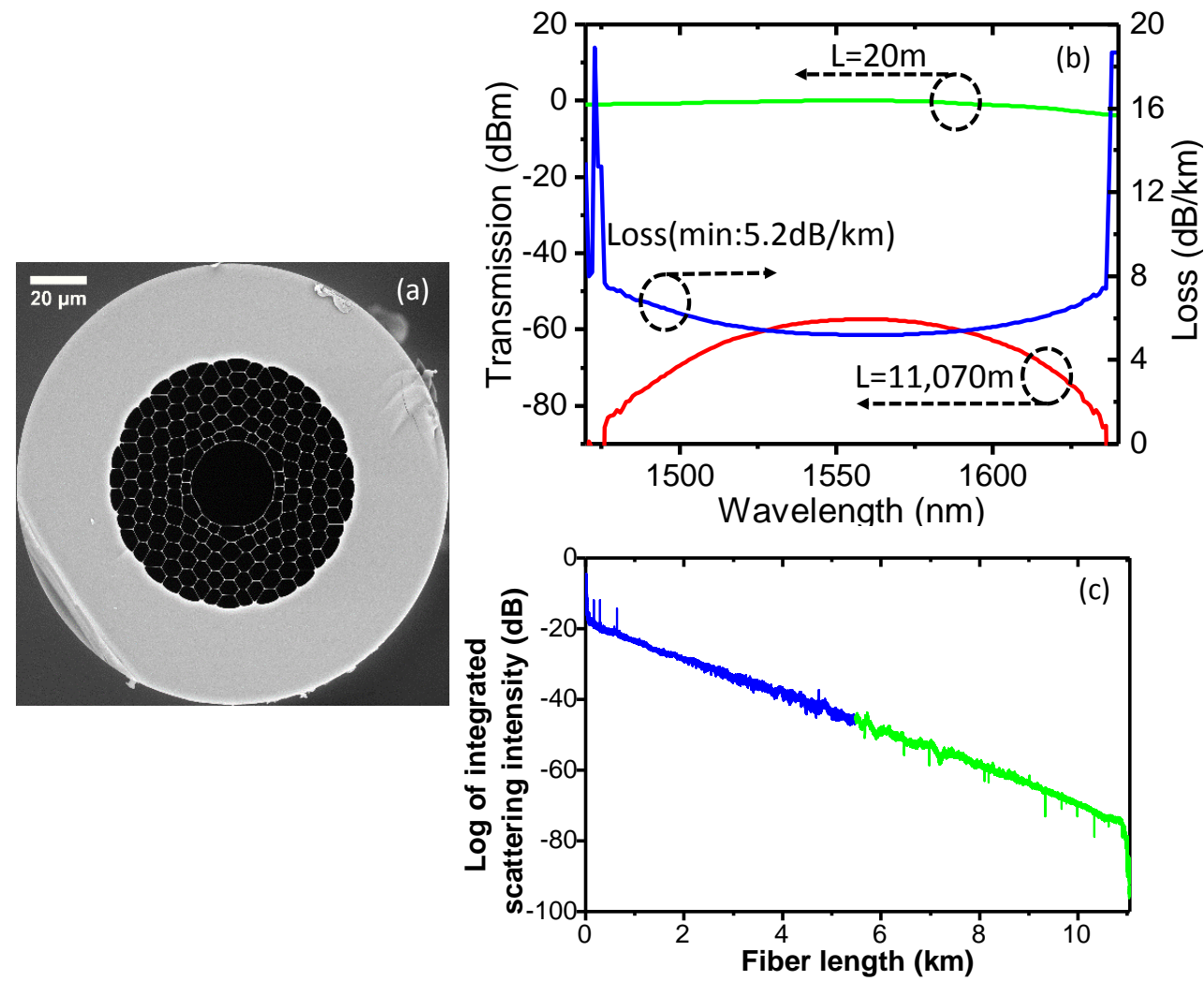

Fig. 1: (a) SEM of the HC-PBGF; (b) the cutback loss measurement results, while noise peaks in both edges of the loss curve were due to limited source power and OSA dynamic range. A more than $200 \mathrm{~nm} 3 \mathrm{~dB}$ bandwidth was determined by anther cutback measurement in a short section of the fiber; (c) side scattering results demonstrating its longitudinal uniformity along the whole fiber length. The blue and green traces represent measurements obtained when launching from opposite directions of the whole $11 \mathrm{~km}$ span. The green one is plotted reversed for graphic purposes. 


\section{BENDING LOSS OF THE HC-PBGF}

The bending loss was measured by using a white light source (Bentham WLS100) and optical spectral analyzer (Ando AQ6315). A large mode area fiber was utilized for optimum input coupling into the HC-PBGF. From previous observations, this approach ensures efficient and selective coupling to the fundamental mode of the HC-PBGF, although lower intensity higher-order modes inevitably exist. For all measurements, we then chose to fine tune the input coupling so as to maximize the overall output power; input launch conditions are thus consistent for all the measurements. Particular care was taken to ensure that the source intensity and coupling condition were stable during all the measurements.
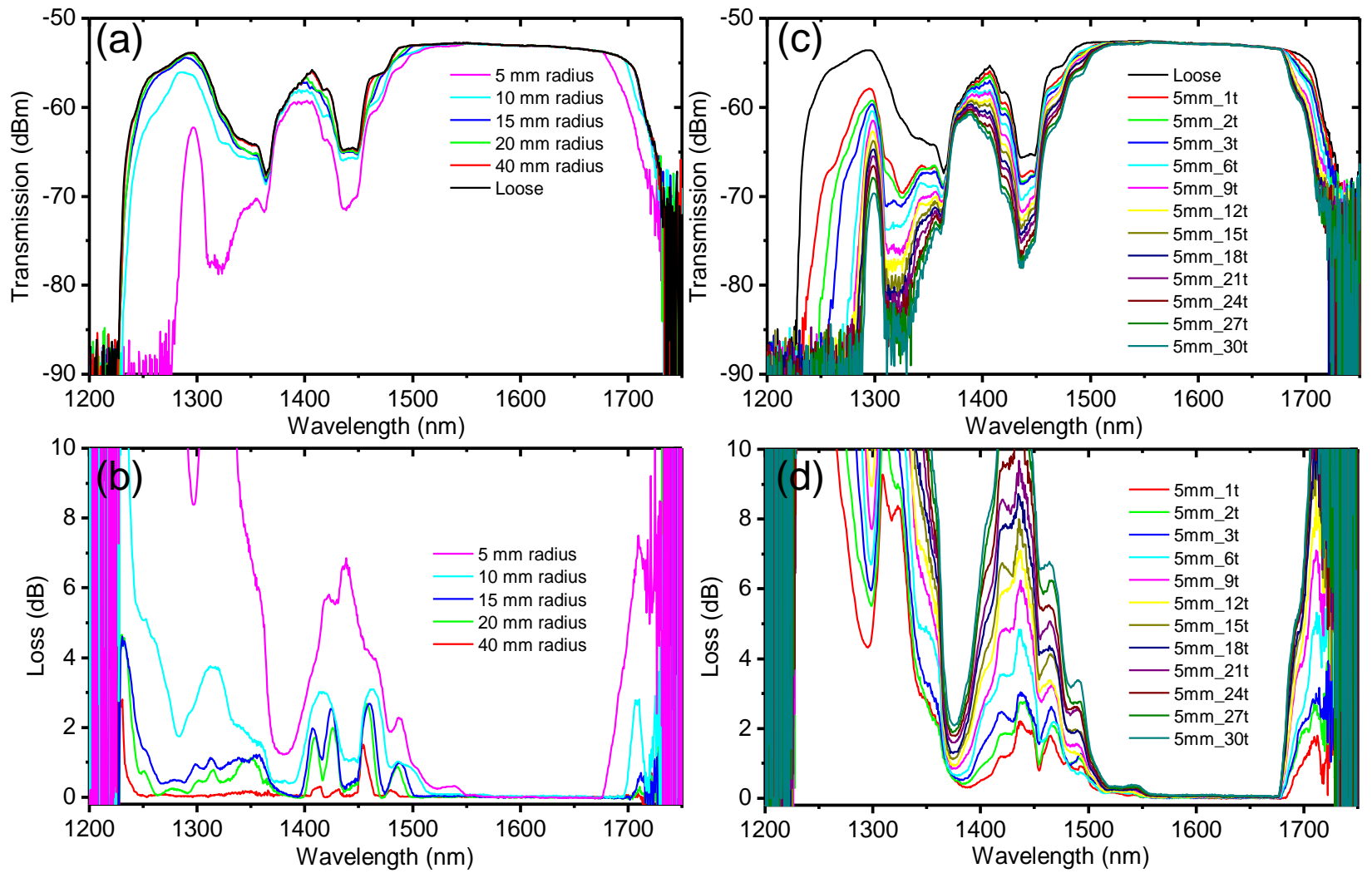

Fig. 2: Fiber transmission spectra (a) and bend loss spectra (b) for 10 turns of decreasing bend radii; sample length used for the experiment is $60 \mathrm{~m}$. Transmission (c) and bend loss (d) measured for a fixed bend radius value of $5 \mathrm{~mm}$ and gradually increasing the number of turns.

Bending loss was measured in two ways. Firstly, we imposed a fixed number of 10 turns but with different radii (40mm, $20 \mathrm{~mm}, 15 \mathrm{~mm}, 10 \mathrm{~mm}$, and $5 \mathrm{~mm}$ ). The corresponding transmission spectra were recorded and compared to the transmission spectrum of the fiber in a "loose" or "unbent" condition (the fiber was loosely coiled with a diameter of $\sim 32 \mathrm{~cm}$ ). The results are shown in Fig 2(a) and 2(b) and highlight a monotonic increase of the bend loss as the bend radius is reduced. We then fixed the bend radius to the smallest value measured in the previous series, i.e. $5 \mathrm{~mm}$, and increased the number of turns (i.e. the length of fiber bent) gradually from a single turn to a maximum of 30 turns. The transmission spectra were recorded and again compared to the unbent spectral transmission - the results are shown in Fig. 2(c) and 2(d).

The effect of bending on this fiber can be divided broadly into two regimes: over the first region, which coincides with a substantial portion of the lowest loss transmission window of the fiber, the fiber loss is negligibly affected by bending we term this the bend insensitive region; outside the bend insensitive region, i.e. close to the bandgap edges and to the surface modes anti-crossing points, the loss is increased through bending. This is the bend sensitive region. 
We first focus on the bend insensitive region. As shown in Fig. 2, even in the most extreme condition (5mm diameter, 30 turns), there is negligible bend loss $(<0.1 \mathrm{~dB})$ for wavelengths between $1550 \mathrm{~nm}$ and $1680 \mathrm{~nm}$ in this fiber. These bendresistant region coincides with the low loss region of the fiber, which is obvious by comparing to the loss measurement shown in Fig 1(b). If consider the large mode field diameter $(\sim 22 \mu \mathrm{m})$ of the fiber, this level of bend-resistance is even remarkable, which is much superior to that of a standard single mode fiber under similar bending conditions.

We then studied the bending sensitive region in detail. We observed that bending caused a "red" shift of the short wavelength bandgap edge to longer wavelengths, which is similar to what was reported by Hansen et al ${ }^{4}$. By comparing the curves for "unbent" and 5mm bend radius in Fig 2(a) such shift - in this case approaching 50nm, is very obvious. By comparison the long wavelength edge appears to be more robust, though a weaker "blue" shift (i.e. to short wavelength) is also observed. The net effect of the edge shifts is a reduction of the transmission bandgap, though the region of lowest loss in our fiber is relatively unaffected. The effect can be explained with an increased coupling from the fundamental air guided mode to cladding modes as the fiber is subject to increasingly tighter bends. A similar behavior to what described here has been observed in an all-solid photonic bandgap fiber ${ }^{7}$. We suggest that the underling physics should be the same in both fibers despite obvious structural differences. Unsurprisingly, the region where surfaces modes are located are also affected by bending. An important feature of the transmission of the bent fiber and also shown in the bend loss curves is the presence of distinctive loss steps located at wavelengths from the low loss region $(\sim 1550 \mathrm{~nm})$ to the surface mode anti-crossing peak at $\sim 1425 \mathrm{~nm}$. At least three different steps can be identified: 1450-1480, 1480-1510 and 1510-1540nm. These loss steps can be found in other spectral regions, although they are relatively less obvious compare to those between $1425 \mathrm{~nm}$ and $1550 \mathrm{~nm}$.

In order to understand these loss plateaus, a high fidelity reproduction of the fiber cross-section from its SEM image was input into a finite element solver and its modal properties were simulated ${ }^{8}$. The map showing the effective refractive indices for the closet 40 modes to the air line (including the first 5 core-guided LP mode groups as highlighted) are shown in Fig. 3(b). It can be observed that both the position and width of the photonic bandgap obtained by simulation agree very well with the experiment results in the unbent regime, which can be seen by comparing Fig 3(b) to Fig 2 (a) or Fig 2 (c). In particular, our model is capable of predicting very accurately the position of the surface mode anticrossings, which divide the transmission bandgap into three sub-windows.

To assist discussion, we directly compare the modal map to the spectral features in the bend loss spectrum recorded for a bend of 5mm bend radius and 30 turns, shown in Fig 3 (a) and (b). As can be seen from Fig 3 (b), the fundamental mode ( $\mathrm{LP}_{01}$ mode group) has the highest effective index and exists over the widest range of wavelengths, being the only coreguided mode near the short wavelength edge of each of the three sub-windows. Higher order modes have progressively lower effective indices and thus exist over narrower windows centered at increasingly longer wavelengths. At the long wavelength edge, the situation reverses, in that the fundamental mode is the first to interact with cladding or surface modes and thus to experience high loss, followed by higher-order modes. The various air guided modes in a HC-PBGF thus have a short wavelength cutoff, a concept analogous to conventional step-index fibers (although it is a long wavelength cutoff in that case) ${ }^{9,10}$. It is well known, for a step index single mode fiber, that several loss peaks will be presented at short wavelengths (where the single mode fiber no longer "single mode") if it is bent to a certain radius. This feature is commonly used to determine the cutoff wavelength of a single mode fiber ${ }^{10}$. Similar situation happens in the HC-PBGF. However, the signature of this effect in these fibers appears in the form of the decreasing loss steps from 1450 to $1540 \mathrm{~nm}$ in Fig 3 (a), with step edges corresponding to air guided modes' cutoff wavelengths. This is due to the unique short wavelength cutoff feature, as we have just discussed and the fact that these cutoff wavelengths for higher order modes are much closer in HC-PBGFs than in a step index fiber. Furthermore, the increased bend sensitivity near the short wavelength side of a bandgap ${ }^{7,9}$ in a HC-PBGF make this staircase effect more pronounced. It is apparent that by exploiting this feature, bending test offer a direct method for determining different core guided modes' cut-off wavelengths (including both the fundamental mode and other higher-order modes) in a HC-PBGF. This is useful, for example, when strict single mode operation is required, as in many applications where modal quality is of great importance. Indeed there will be a narrow band over which a HC-PBGF operates effectively as a single mode fiber (around 1450nm-1480nm in this particular case). 


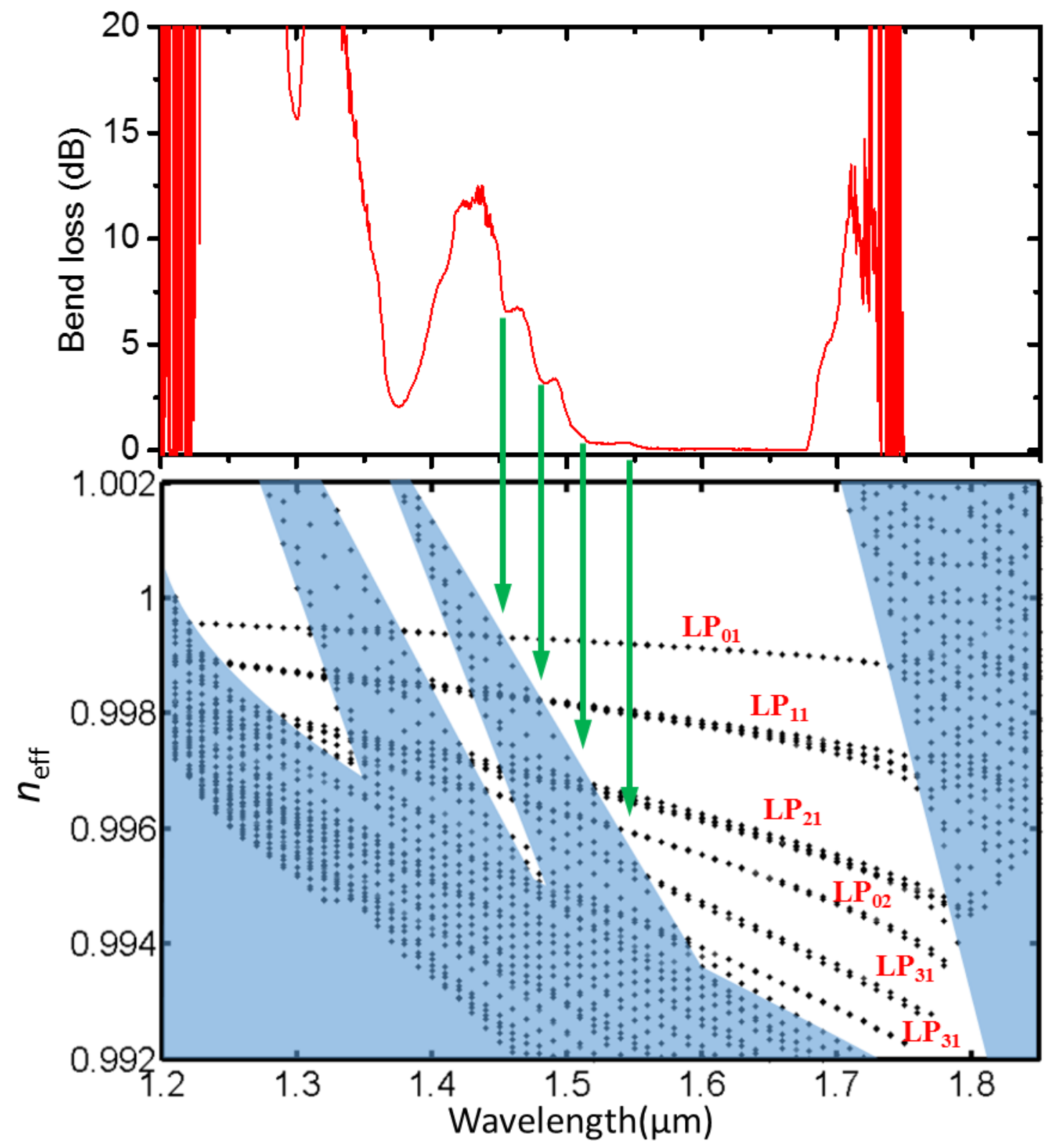

Fig. 3: Comparison between (a) the bend loss spectrum measured for a $5 \mathrm{~mm}$ bend radius and 30 turns, and (b) Simulated mode effective refractive index maps for the fiber in this study. The shaded areas represent areas outside the bandgap (modes only guided in the cladding) and surface modes within the bandgap, where pure core modes are equally not guided. It can be seen that there are two groups of surfaces modes in the bandgap, which separate it to three sub-sections. More importantly, the map shows the cutoff wavelengths of the various core guided modes, i.e. wavelengths at which those modes begin interacting with the surface/cladding modes and thus become lossier.

\section{CONCLUSION}

An in depth understanding of macro-bending loss in HC-PBGFs is important for their long haul data transmission tests and sensing applications. We used the differential transmission loss measurements to explore bend effects in such fiber. There are some features that can be observed at its bending-sensitive region. For example, there is a "red shift" at the 
short wavelength, while this shift becomes "blue" when comes to the long wavelength bandgap edge. Based on the bending loss spectra, it is possible to identify the cutoff wavelengths for different air guiding modes in a HC-PBGF. Despite these features, the most important and useful part of a HC-PBGF transmission window is the bending-insensitive section. Our results show that the fiber is substantially bend-insensitive over a bandwidth region of $\sim 130 \mathrm{~nm}$ at the center of the transmission bandgap, with negligible loss $(<0.1 \mathrm{~dB})$ for bending radii down to $5 \mathrm{~mm}$ (30 turns). This uniquely positions HC-PBGFs as superior media for many applications requiring tight bending.

\section{REFERENCES}

[1] F. Poletti, M. N. Petrovich and D. J. Richardson, "Hollow-core photonic bandgap fibers: Technology and applications," Nanophotonics, vol. 2, no. 6, pp.315 -340 (2013).

[2] F. Poletti, N. V. Wheeler, M. N. Petrovich, N. K. Baddela, E. Numkam Fokoua, J. R. Hayes, D. R. Gray, Z. Li, R. Slavík, and D. J. Richardson, "Towards high-capacity fibre-optic communications at the speed of light in vacuum," Nat. Photonics, vol. 7, no. 4, pp. 279-284 (2013).

[3] Y. Chen, Z. Liu, S. R. Sandoghchi, G. T. Jasion, T. Bradley, E. Numkam Fokoua, J. Hayes, N. V. Wheeler, D. R. Gray, B. J. Mangan, R. Slavík, F. Poletti, M. N. Petrovich, and D. J. Richardson, " Multi-kilometer long, longitudinally uniform Hollow Core Photonic Bandgap Fibers for broadband low latency data transmission," J. Lightwave Technol., vol. 34, no.1, pp. 104-113 (2016).

[4] T. P. Hansen, J. Broeng, C. Jakobsen, G. Vienne, H. R. Simonsen, M. D. Nielsen, P. M. W. Skovgaard, J. R. Folkenberg, and A. Bjarklev, "Air-Guiding Photonic Bandgap Fibers: Spectral Properties, Macrobending Loss, and Practical Handling," J. Lightwave Technol. 22, pp.11-15(2004).

[5] Y. Chen, Z. Liu, S. R. Sandoghchi, G. T. Jasion, T. Bradley, E. Numkam Fokoua, J. Hayes, N. V. Wheeler, D. R. Gray, B. J. Mangan, R. Slavík, F. Poletti, M. N. Petrovich, and D. J. Richardson, "Demonstration of an $11 \mathrm{~km}$ Hollow Core Photonic Bandgap Fiber for Broadband Low-latency Data Transmission," in Optical Fiber Communication Conference Post Deadline Papers, OSA Technical Digest (online) (Optical Society of America, 2015), paper Th5A.1.

[6] S.R.Sandoghchi, M.N.Petrovich, D.R.Gray, Y. Chen, N.V.Wheeler, T.D.Bradley, N.H.L.Wong, G.Jasion, J.R.Hayes, E.Numkam Fokoua, M.Botelho Alonso, S.M.Abokhamis Mousavi, D.J.Richardson, F.Poletti, "Optical side scattering radiometry for high resolution, wide dynamic range longitudinal assessment of optical fibers," Opt Express 23(21), pp.27960-27974(2015).

[7] T. A. Birks, F. Luan, G. J. Pearce, A. Wang, J. C. Knight, and D. M. Bird, "Bend loss in all-solid bandgap fibres," Opt. Express 14, pp.5688-5698 (2006).

[8] E.Numkam Fokoua, S.R.Sandoghchi, Y. Chen, G.T.Jasion, N.V.Wheeler, N.K.Baddela, J.R.Hayes, M.N.Petrovich, D.J.Richardson, F.Poletti, "Accurate modelling of fabricated hollow-core photonic bandgap fibers," Opt Express Vol.23(18), pp. 23117-23132 (2015).

[9] M. J. F. Digonnet, H. K. Kim, G. S. Kino, and S. Fan, "Understanding Air-Core Photonic-Bandgap Fibers: Analogy to Conventional Fibers," J. Lightwave Technol. 23, pp. 4169-4177(2005).

[10] J. A. Buck, Fundamentals of Optical Fibers, Wiley (2004). 